brief advice, 16-19 extended brief advice, $>20$ specialist referral offered). 13/68 (19\%) patients with no documented alcohol history had an AUDIT score of $>7,3$ of whom had an AUDIT score of $>20.2 / 61$ patients with an. alcohol history documented as 'no alcohol' had an AUDIT score $>7$. Chest pain was the most common $(n=7)$ presenting complaint for patients with an AUDIT score $>7$, followed by shortness of breath $(n=4)$. gGT and MCV were poor predictors of hazardous alcohol consumption. The FAST questionnaire achieved $94 \%$ sensitivity and $100 \%$ specificity in detecting patients with hazardous alcohol consumption judged by the AUDIT score. Furthermore, FAST was considered easier and quicker to use.

Conclusion Hazardous alcohol consumption is frequently under recognised, leading to missed opportunities for effective interventions that may reduce harm. Patients admitted with chest pain are not identified as being at high risk in the NICE guidance, but contain the highest number of hazardous drinkers in our study. Abbreviated questionnaires are accurate and easily used, and should be incorporated into an admission clerking of all presenting complaints.

Competing interests None.

Keywords alcohol misuse.

\section{OC-096 IMPROVING THE ACUTE MEDICAL TAKE: THE IDENTIFICATION OF HAZARDOUS ALCOHOL CONSUMPTION}

doi:10.1136/gut.2011.239301.96

A J Ball,, D Leaning, D A Elphick Department of Gastroenterology, Chesterfield Royal Hospital, Chesterfield, UK

Introduction Hazardous alcohol consumption is a growing public health problem. Recent NICE guidance suggests systematic identification of patients who misuse alcohol through the use of validated screening questionnaires such as the World Health Organisation developed AUDIT (Alcohol Use Disorders Identification Test) tool. Studies have shown brief targeted interventions in at risk groups to be cost effective in reducing readmission rates and the amount of alcohol consumed. We aimed to see how effectively patients drinking hazardous amounts of alcohol were being identified on our medical admissions unit and to compare AUDIT with the shorter FAST (Fast Alcohol Screening Tool).

Methods A randomly selected cohort of medical patients was prospectively recruited on the day of their admission to Chesterfield Royal Hospital, between January 2010 and March 2010. Case note review for demographic data, documentation of alcohol history, MCV, gGT, and presenting complaint was performed. The AUDIT and FAST questionnaires were then performed with the patient.

Results 188 patients (age range 17 - 100, median age 70) were included. AUDIT scores ranged 0-40. 156 (83\%) had score $£ 7$; 21 (11\%) had score 8-15 (hazardous); 4 (2\%) had score 16-19 (harmful) and 7 (4\%) had score 20 (possible dependence). Only $1 / 32$ patients with an alcohol score of $>7$ had appropriate advice offered and documented according to their AUDIT score (8-15 